\title{
From 6LoWPAN to 6Lo: Expanding the Universe of IPv6-Supported Technologies for the Internet of Things
}

Article in IEEE Communications Magazine · December 2017

DOI: 10.1109/MCOM.2017.1600534

CITATIONS

0

4 authors, including:

\section{Carles Gomez}

Universitat Politècnica de Catalunya

77 PUBLICATIONS 1,153 CITATIONS

SEE PROFILE
READS

178
Carsten Bormann

Universität Bremen

64 PUBLICATIONS 1,303 CITATIONS

SEE PROFILE 
Author's pre-print, accepted for publication in the IEEE Communications Magazine (2017-7-13).

copyright 2017 IEEE. Please refer to the final version once published.

Personal use of this material is permitted. Permission from IEEE must be obtained for all other users, including reprinting/ republishing this material for advertising or promotional purposes, creating new collective works for

resale or redistribution to servers or lists, or reuse of any copyrighted components of this work in other works.

\title{
From 6LoWPAN to 6Lo: Expanding the Universe of IPv6-Supported Technologies for the Internet of Things
}

\author{
Carles Gomez ${ }^{1}$, Josep Paradells ${ }^{2}$, Carsten Bormann ${ }^{3}$, Jon Crowcroft ${ }^{4}$ \\ ${ }^{1}$ Universitat Politècnica de Catalunya (UPC) \\ ${ }^{2}$ Universitat Politècnica de Catalunya (UPC)/i2CAT Foundation \\ ${ }^{3}$ Universität Bremen TZI \\ ${ }^{4}$ University of Cambridge \\ E-mail: \{carlesgo,teljpa\}@entel.upc.edu,cabo@tzi.org, jon.crowcroft@cl.cam.ac.uk
}

\begin{abstract}
Leveraging 6LoWPAN, the IETF 6Lo working group has targeted adaptation of IPv6 over a new generation of communication technologies for the IoT. These comprise Bluetooth LE, ITU-T G.9959, DECT ULE, MS/TP, NFC, IEEE 1901.2, and IEEE 802.11ah. This paper comprehensively analyzes the 6Lo technologies and adaptation layers, giving the motivation for critical design decisions, highlighting crucial aspects for performance, and presenting main challenges.
\end{abstract}

\section{Introduction}

The Internet of Things (IoT) is an emerging networking paradigm whereby daily life objects, some of them equipped with sensors or actuators, are connected to the Internet. The IoT vision promises a revolution, leading to substantial improvement in global sustainability, efficient resource management, enhanced productivity and increased human life quality, by enabling innovative applications in a wide variety of trending domains. Examples of the latter include smart cities, home and building automation, smart factories, smart grid, remote health, intelligent transportation systems, etc.

Making the IoT become a reality is a multiagent effort comprising advances in various technical fields and the involvement of academia, industry, public administration and standards development organizations. To enable the aforementioned IoT applications, a plethora of inexpensive, network-capable IoT devices are being deployed worldwide. In order to provide Internet connectivity to the vast number of IoT devices, estimated as a few tens of billions by year 2020 [1], IPv6 is the optimal protocol given its large set of available addresses, tools for unattended operation, and intrinsic interoperability. However, IPv6 was designed for resource-rich networking scenarios (e.g. Ethernets), whereas common IoT network environments are significantly more limited. The latter comprehend devices with severe energy, memory, processing, and communication constraints. Such devices use wireless (or wired) links characterized by low data rate, short data unit length, high bit error rate and variable link quality. Therefore, adaptation functionality is required to support and optimize IPv6 over constrained-node networks. Such functionality is usually abstracted as an adaptation layer, that is, a protocol stack layer inserted between IPv6 and a target technology, designed to efficiently enable IPv6 over that technology (Figure 1.a). An adaptation layer may provide lightweight encoding formats (e.g. IPv6 header compression), support for data transport (e.g. 
fragmentation and reassembly over technologies with short frame payload, multihop data delivery for mesh topologies, etc.), and energy-frugal network parameter configuration.

a)

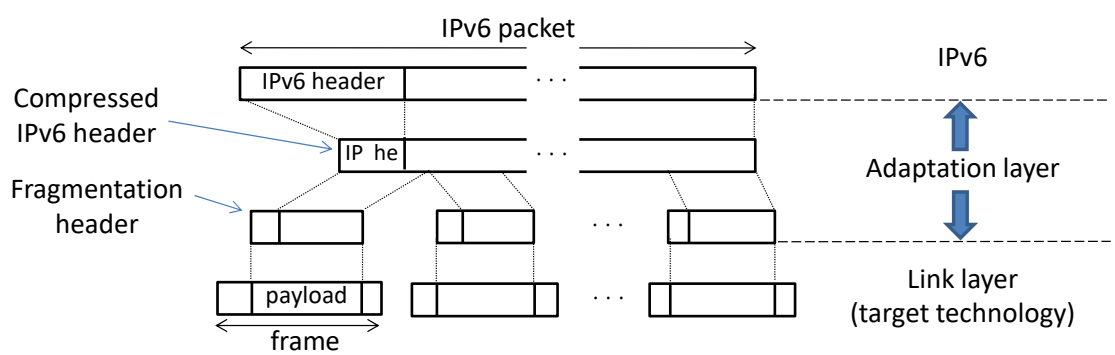

b)

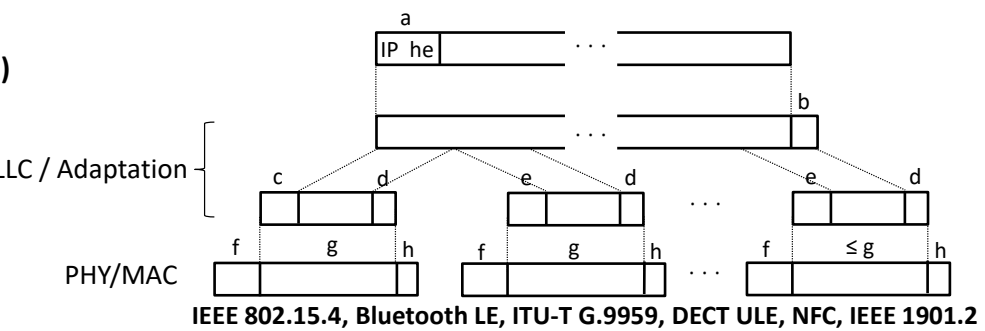

c)

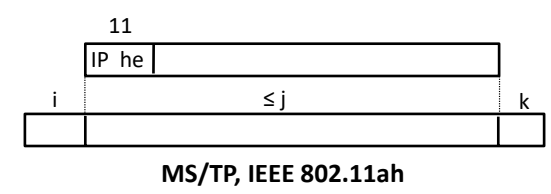

PHY/MAC

MS/TP, IEEE 802.11ah

\begin{tabular}{|c|c|c|c|c|c|c|}
\cline { 2 - 7 } \multicolumn{1}{c|}{} & \multicolumn{7}{c|}{ Field size (bytes) } \\
\hline Field & IEEE 802.15.4 & Bluetooth LE & ITU-T G.9959 & DECT ULE & NFC & IEEE 1901.2 \\
\hline a & 11 & 7 & 11 & 7 & 11 & 11 \\
\hline b & 0 & 0 & 0 & 2 & 0 & 0 \\
\hline c & 4 & 8 & 4 & 4 & 7 & 0 \\
\hline d & 0 & 0 & 2 & 0 & 0 & 0 \\
\hline e & 5 & 6 & 5 & 4 & 7 & 0 \\
\hline $\mathbf{f}$ & 29 & 15 & 34 & 12 & 5 & 40 \\
\hline g & 102 & 27 & 158 & 38 & 128 & 215 \\
\hline h & 2 & 3 & 2 & 2.5 & 2 & 2 \\
\hline
\end{tabular}

\begin{tabular}{|c|c|c|}
\cline { 2 - 3 } \multicolumn{1}{c|}{} & \multicolumn{2}{c|}{ Field size (bytes) } \\
\hline Field & MS/TP & IEEE 802.11ah \\
\hline i & 8 & 30 \\
\hline j & 1500 & 7951 \\
\hline k & 5 & 4 \\
\hline
\end{tabular}

Figure 1.a) An example of adaptation layer functionality; b) IPv6 datagram encapsulation over 6 Lo technologies, fragmentation needed; c) IPv6 datagram encapsulation over 6Lo technologies, fragmentation not needed.

In late 2004, the IETF IPv6 over Low power Wireless Personal Area Networks (6LoWPAN) Working Group (WG) started to produce the adaptation layer to support IPv6 over IEEE 802.15.4, the de-facto low-rate, low-power wireless radio interface. The 6LoWPAN adaptation layer [2] was completed by the end of 2012, and thus the 6LoWPAN WG was subsequently closed. However, the panoply of communication technologies for constrained devices had been (and still is) growing steadily. Therefore, the need to extend IPv6 support to a new generation of wireless and wired communication technologies for the IoT was identified. The IETF IPv6 over Networks of Resource-constrained Nodes (6Lo) WG was created in 2013 with the main goal of carrying out this crucial work, leveraging 6LoWPAN. As of the writing, the 6Lo WG has targeted IPv6 adaptation for a wide and diverse set of communication technologies 
(hereinafter, 6Lo technologies), ranging from generic-purpose to application-specific solutions, which comprises Bluetooth Low Energy (Bluetooth LE), ITU-T G.9959, DECT Ultra Low Energy (DECT ULE), Master-Slave/Token-Passing (MS/TP), Near Field Communication (NFC), IEEE 1901.2, and IEEE 802.11ah. Figure 2 summarizes the timespan of the 6LoWPAN and 6Lo WGs activity, and the chronology of their produced specifications.

The work carried out by the 6Lo WG, which comprises both completed and on-going standardization items, is expected to connect several billion devices to the Internet (of Things). However, it has only been considered to a very limited extent in the literature. For the first time to our best knowledge, this paper provides a comprehensive analysis of the 6Lo technologies and adaptation layers. The remainder of the paper is organized as follows. Section 2 overviews the wireless and wired interfaces targeted by the 6LoWPAN and 6Lo WGs. Section 3 describes the main 6LoWPAN and 6Lo components, and discusses the design solutions adopted to enable and optimize IPv6 over the 6 Lo technologies. Section 4 presents main 6 Lo challenges. Finally, section 5 concludes the paper.

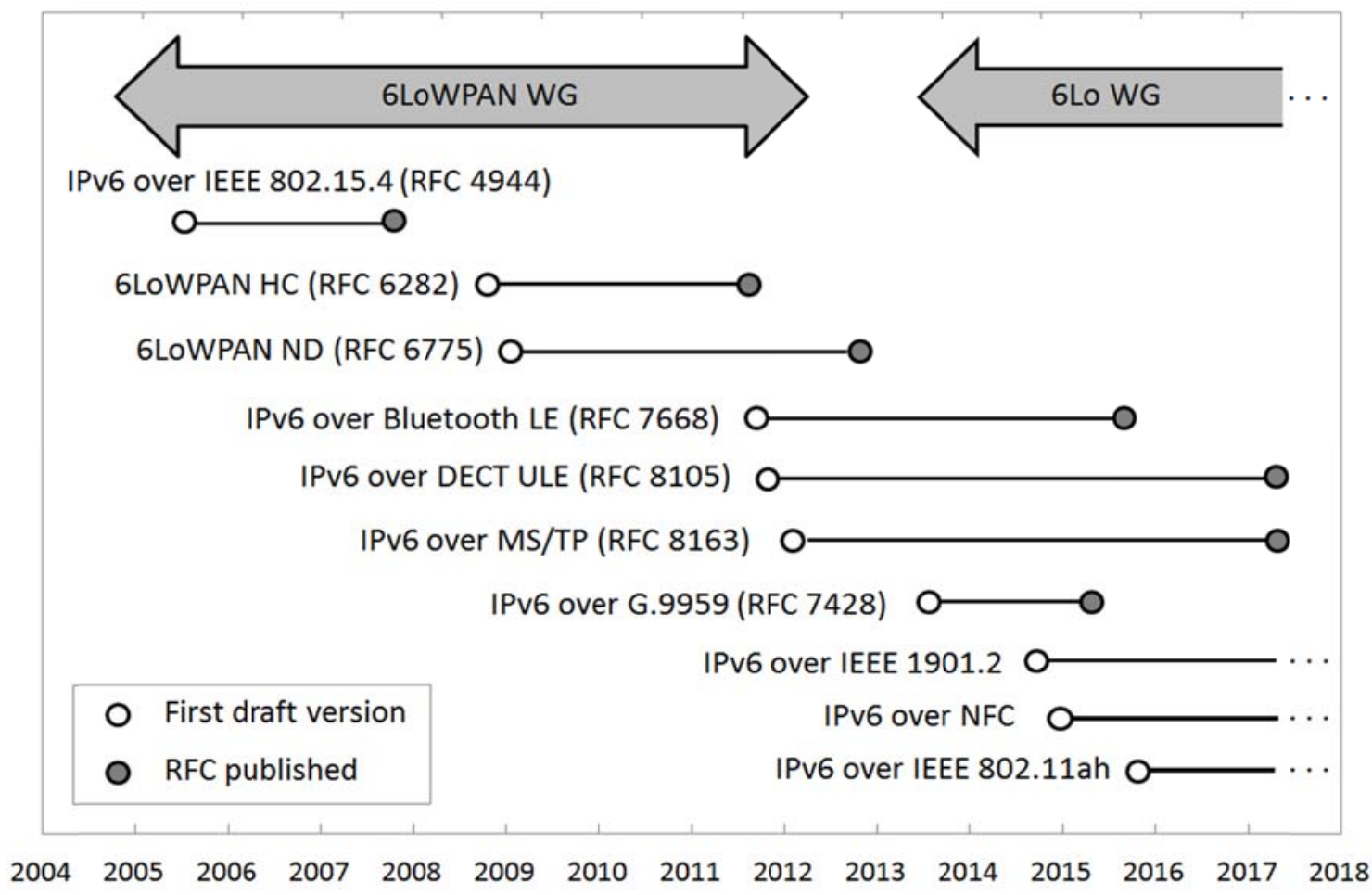

Figure 2. Chronology and timespan of the 6LoWPAN and 6Lo WGs adaptation layer specifications and activity.

\section{Wireless and wired technologies for the Internet of Things}

This section introduces and discusses the wireless and wired technollogies for which IPv6 support has been or is being developed by the 6LoWPAN and 6Lo WGs. The main 
features of each technology, highlighting those especially relevant for IPv6 adaptation, are shown in Table I.

\subsection{IEEE 802.15.4}

IEEE 802.15.4 is the seminal standard defining physical layer (PHY) and Medium Access Control layer (MAC) functionality for low-power wireless applications. The original version of this standard was published in 2003, constituting a milestone in its application space. Shortly after, the IETF established the 6LoWPAN WG with the aim of enabling IPv6 support over IEEE 802.15.4 networks. Since then, several IEEE 802.15.4 amendments have been released, and a plethora of on-going amendment efforts for specialized environments are currently in progress. Remarkably, a specific IETF WG called 6TiSCH was established in 2013 to define the mechanisms and architecture to enable IPv6 over Time Slotted Channel Hopping (TSCH), a salient mode of IEEE 802.15.4e [3]. This intense activity is a sign of IEEE 802.15.4 technology's good health.

\subsection{Bluetooth LE / Bluetooth Smart}

Bluetooth LE, also marketed as Bluetooth Smart, is the hallmark feature in the Bluetooth 4.0 specification, published in 2010. Bluetooth LE is a protocol stack that was designed in order to enable multi-year operation for battery-powered devices such as sensor nodes. Most smartphones are currently being manufactured with Bluetooth LE support, therefore the potential space for Bluetooth LE is counted in billions of units. With Bluetooth LE, the smartphone may act as a gateway able to collect data from sensors, wearables, and other consumer electronics devices, and to send those data to the Internet (e.g. via $4 \mathrm{G}$ or $\mathrm{Wi}-\mathrm{Fi}$ ). Bluetooth $\mathrm{LE}$ extensions have been recently provided in subsequent Bluetooth specifications. The functionality required to enable IPv6 over Bluetooth LE comprises the IPv6 over Bluetooth LE specification, recently published as RFC 7668, and the Bluetooth SIG Internet Protocol Support Profile (IPSP) [4]. RFC 7668 has served as a model for 6Lo specifications over technologies that define simple network topologies such as star or point-to-point.

\subsection{ITU-T G.9959}

Z-Wave is a protocol stack developed more than a decade ago by ZenSys (now, Sigma Designs) for wireless home automation. Z-Wave has gained significant market presence in this domain, in fact having been available in products earlier than competing technologies such as ZigBee [5]. Born as a proprietary solution, Z-Wave PHY and MAC layers were first standardized by the ITU-T in 2012 as the G.9959 specification. The first adaptation layer specification produced by the 6Lo WG, published as RFC 7428, defines the adaptation of IPv6 over ITU-T G.9959 networks. These standardization efforts extend the Internet connectivity possibilities of this technology beyond existing Z-Wave-to-IP (Z/IP) protocol translation gateways. 


\subsection{DECT ULE}

The Digital Enhanced Cordless Telecommunications (DECT) radio interface has been used for more than two decades for wireless telephony and data applications in indoor scenarios. Recently, a new variant of this technology called DECT ULE has been standardized by the ETSI to enable low-power wireless applications. DECT ULE introduces MAC layer changes to the original DECT standard, while it retains PHY compatibility. Therefore, DECT ULE has significant potential given the strong DECT deployment base, especially in the home area. The 6Lo WG has created RFC 8105, a specification to support IPv6 over DECT ULE.

\subsection{MS/TP}

MS/TP is a MAC layer mechanism defined for the RS-485 PHY. These MAC and PHY layers are a subset of the options defined in BACnet, an ANSI/ASHRAE standard extensively used in the building automation domain. Remarkably, RS-485 defines a Shielded Twisted Pair (STP) wired, low-error rate multidrop bus. Furthermore, devices in MS/TP networks have a continuous power source. On the other hand, such networks offer limited data rates, and MS/TP devices are often constrained in processing power and memory; to benefit from IPv6, the IETF 6Lo WG has produced a specification (RFC 8163) to adapt IPv6 over MS/TP networks, even if the latter are relatively different environments compared with those of other communication technologies for constrained devices.

\subsection{NFC}

Near Field Communication (NFC) comprises a set of standard technologies for shortrange (i.e. below $20 \mathrm{~cm}$ ) wireless communication. NFC is available in a variety of devices, including tags and the main smartphone platforms. Since sales of the latter are currently being counted in hundreds of millions per year, the potential of NFC is enormous. Its short range provides intrinsic security properties, as in fact, electronic payment has become a popular application for this technology. When two NFC endpoints are powered, a peer-to-peer NFC mode can be used. A specification to support IPv6 over the peer-to-peer mode of NFC is being developed by the 6Lo WG.

\subsection{IEEE 1901.2}

Power Line Communication (PLC) has been used for decades for home automation, telemetry and broadband Internet access. IEEE 1901.2 is a PLC standard approved in 2013 (amended in 2015), intended for smart grid applications, that defines PHY and MAC functionality for narrowband communications. IEEE 1901.2 is designed to overcome harsh conditions such as adverse Signal-to-Noise-Ratio (SNR) and variable characteristics (e.g. line impedance) in space, time and frequency. In fact, IEEE 1901.2 links share a significant degree of commonality with low-power wireless links, such as high bit error rate. A specification to support IPv6 over IEEE 1901.2 has been proposed 
in the 6Lo WG, although the IEEE 1901.2 specification also describes how to configure an IPv6-based IEEE 1901.2 multihop network.

\subsection{IEEE 802.11ah}

IEEE 802.11ah is an IEEE 802.11 amendment whose standardization process is expected to be completed in the near future. It addresses the lack of low-power support for sensor networks in the IEEE 802.11 standard. Target applications of IEEE 802.11ah comprise metering, and backhaul links for data collected by sensors. IEEE 802.11ah is favored by the massive popularity of $\mathrm{Wi}-\mathrm{Fi}$, but it cannot make use of currently deployed access points, since IEEE 802.11ah is not backwards compatible with previous versions of IEEE 802.11. As of the writing, IEEE 802.11 ah has been the most recent one to join the set of technologies for which IPv6 support is being developed by the $6 \mathrm{Lo} \mathrm{WG}$.

\subsection{Discussion}

Most of the introduced technologies are wireless, which provides flexibility and low deployment cost. Nevertheless, wireless technologies are subject to interference and radio propagation issues. Even if IEEE 1901.2 is wired, it also has low installation cost, since it reuses existing power grid lines, and naturally suffers interference due to coexistence with the electricity signal. In contrast, MS/TP uses a high-quality field bus.

Supported network topologies range from simple point-to-point (NFC) or star topologies (BLE, DECT ULE, IEEE 802.11 ah) to mesh networks (IEEE 802.15.4, ITUT G.9959, IEEE 1901.2). The latter involve greater complexity to enable end-to-end communication, however they better overcome coverage limitations and offer path diversity. For example, nowadays home automation deployments are mainly based on mesh network technologies. On the other hand, MS/TP, IEEE 1901.2 and IEEE 802.11ah offer greater link range than the rest of technologies. MS/TP has been designed to reach devices throughout large buildings via a shared bus, while the latter two technologies support smart grid and metering use cases, which involve communication between distant devices such as meters and concentrators that aggregate data from several households. Finally, NFC and BLE exploit their widespread presence in smartphones, which transform the smartphone into both an Internet connectivity gateway and a remote control for direct interaction with sensors or actuators.

\section{6LoWPAN and 6Lo: IPv6 over IoT technologies}

In order to enable IPv6 over IEEE 802.15.4 networks, the adaptation layer called 6LoWPAN was developed. Subsequently, 6Lo made use of 6LoWPAN as a basis to support IPv6 over Bluetooth LE, ITU-T G.9959, DECT ULE, MS/TP, NFC, IEEE 1901.2, and IEEE 802.11ah. This section analyses the following fundamental technical elements and mechanisms of 6LoWPAN and 6Lo: locus of the adaptation layer in the protocol stack, routing, addressing, header compression, fragmentation, encapsulation, 
neighbor discovery, and multicast support. Table 1 includes a summary of the main features of the 6LoWPAN and 6Lo adaptation layers.

\subsection{Locus of the adaptation layer in the protocol stack}

When designing a solution to enable IPv6 over a communications technology, a crucial decision is the locus of the adaptation layer in the protocol stack of that technology. Since IPv6 is a network layer protocol, the 6Lo layer must be placed atop a link layer protocol. When a link layer comprises several sublayers (e.g. a MAC sublayer and also a Link Layer Control (LLC) sublayer), one of the sublayers has to be chosen to interface the 6Lo layer. This selection depends on criteria including header overhead, compatibility with previous technology versions, upper layer multiplexing capabilities, and segmentation and reassembly support (the latter avoids the need for fragmentation at the 6Lo layer). Figure 3 shows the IPv6-based protocol stack for each 6Lo or 6LoWPAN technology.

Bluetooth LE, DECT ULE and NFC define complete protocol stacks up to the application layer. When IPv6 is used on top of these, the native upper layer protocols used in each technology are replaced by IP-based upper-layer protocols (e.g. UDP, TCP, etc.) and application-layer protocols (e.g. CoAP [6], HTTP, etc.).

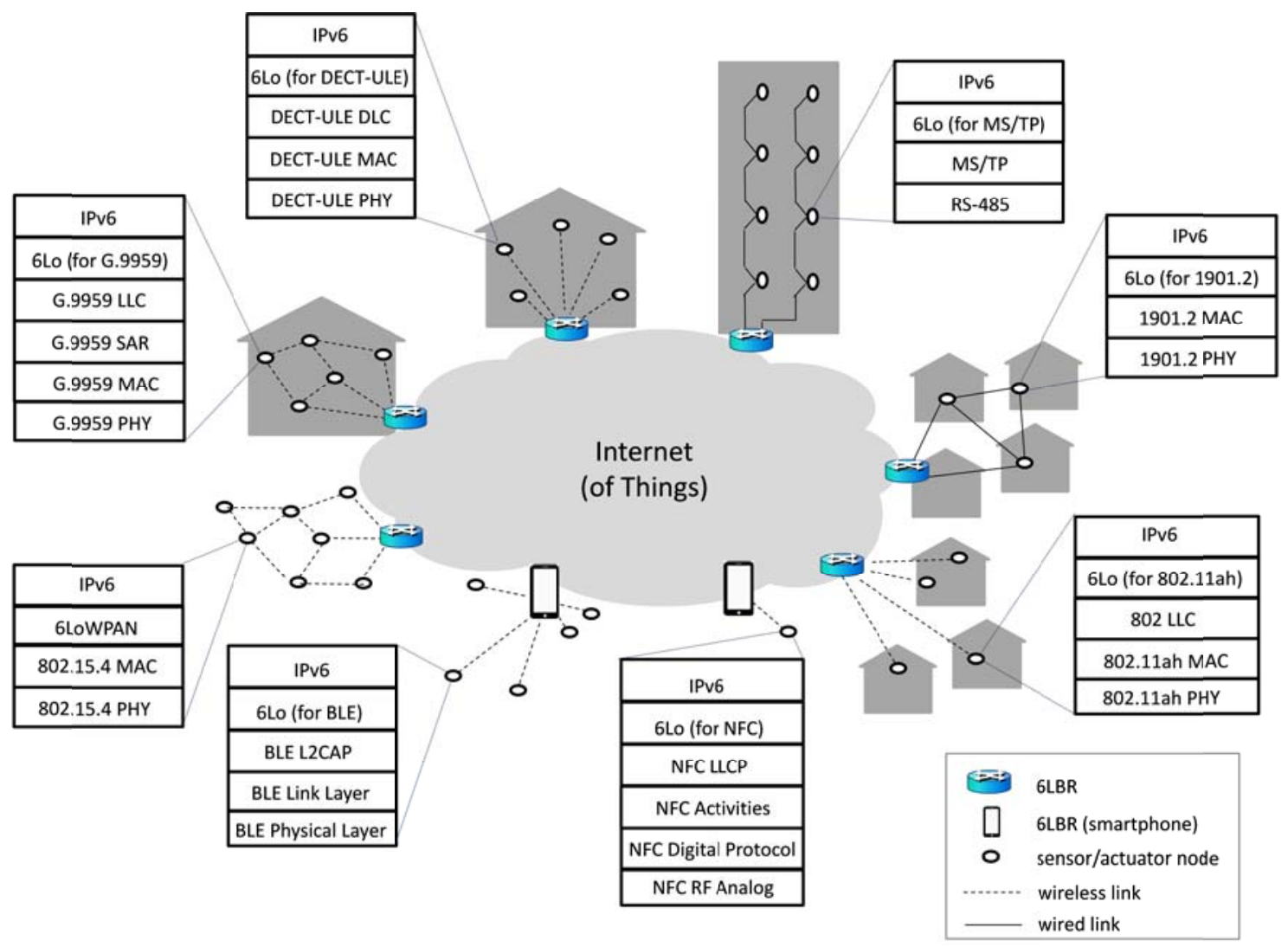

Figure 3. Protocol stack, and example network topology, for each 6Lo/6LoWPAN technology when using IPv6. BLE stands for Bluetooth LE. 


\begin{tabular}{|c|c|c|c|c|c|c|c|c|c|}
\hline & & \multirow{2}{*}{$\begin{array}{c}\text { 6LoWPAN } \\
\text { IEEE } \\
802.15 .4 \\
\end{array}$} & \multicolumn{7}{|c|}{ 6Lo } \\
\hline & & & Bluetooth LE & $\begin{array}{l}\text { ITU-T } \\
\text { G.9959 }\end{array}$ & DECT ULE & MS/TP & NFC & $\begin{array}{l}\text { IEEE } \\
1901.2\end{array}$ & $\begin{array}{c}\text { IEEE } \\
\text { 802.11ah }\end{array}$ \\
\hline \multirow{13}{*}{ Technology } & Medium & Wireless & Wireless & Wireless & Wireless & Wired & Wireless & Wired & Wireless \\
\hline & Frequency band (MHz) & $868 / 915 / 2400$ & 2400 & $868 / 915$ & 1900 & Base-band & 13.56 & $<0.5$ & $<1000$ \\
\hline & Range (m) & $10-100$ & $10-100$ & 100 & $<300$ & 1000 & $<0.2$ & $>1000$ & $<1000$ \\
\hline & Bit rate (kbit/s) & $20 / 40 / 250$ & 1000 & $9.6 / 40 / 100$ & 1152 & 115.2 & $106 / 212 / 424$ & $\leq 500$ & $150-7800$ \\
\hline & $\begin{array}{c}\text { Max. single-frame L2 } \\
\text { payload (bytes) }\end{array}$ & 105 & 23 & 158 & 38 & 2032 & 125 & $\begin{array}{c}215 \\
\text { (worst case) }\end{array}$ & 7951 \\
\hline & ACKs and retries & Optional & Yes & Optional & Yes & No & ACK/NACK & $\begin{array}{l}\text { ACK/NACK } \\
\text { (optional) }\end{array}$ & Yes \\
\hline & MAC mechanism & $\begin{array}{l}\text { CSMA/CA, } \\
\text { TDMA }\end{array}$ & TDMA & CSMA/CA & TDMA & $\begin{array}{c}\text { Token } \\
\text { passing }\end{array}$ & $\begin{array}{c}\text { TDMA link } \\
\text { initialization }\end{array}$ & CSMA/CA & CSMA/CA \\
\hline & Address size (bits) & $16 / 64$ & 48 & 40 & $20 / 40 / 48$ & 8 & 6 & $16 / 64$ & 48 \\
\hline & L2 fragmentation & No & Yes & Yes & Yes & No & Yes & Yes & Yes \\
\hline & Network topology & Star and mesh & Star & Mesh & Star & $\begin{array}{l}\text { Multi-drop } \\
\text { bus }\end{array}$ & $\begin{array}{l}\text { Point-to- } \\
\text { point }\end{array}$ & $\begin{array}{c}\text { Star and } \\
\text { mesh }\end{array}$ & Star \\
\hline & Protocol stack & PHY/Link & PHY to App. & PHY/Link & PHY to App. & PHY/Link & PHY to App. & PHY/Link & PHY/Link \\
\hline & Application & $\begin{array}{l}\text { Generic } \\
\text { purpose }\end{array}$ & $\begin{array}{l}\text { Smartphone- } \\
\text { centric }\end{array}$ & $\begin{array}{c}\text { Home } \\
\text { automation }\end{array}$ & $\begin{array}{c}\text { Home } \\
\text { automation }\end{array}$ & $\begin{array}{l}\text { Building } \\
\text { automation }\end{array}$ & $\begin{array}{c}\text { Contactless } \\
\text { exchange }\end{array}$ & $\begin{array}{c}\text { Smart grid, } \\
\text { home autom. }\end{array}$ & $\begin{array}{l}\text { Sensors, } \\
\text { backhaul }\end{array}$ \\
\hline & $\begin{array}{c}\text { Standardization } \\
\text { organization }\end{array}$ & IEEE & Bluetooth SIG & ITU-T & ETSI & $\begin{array}{c}\text { ANSI/ } \\
\text { ASHRAE }\end{array}$ & NFC Forum & IEEE & IEEE \\
\hline \multirow{8}{*}{$\begin{array}{l}\text { Adaptation } \\
\text { Layer }\end{array}$} & Routing required & Yes & No & Yes & No & No & No & Yes & No \\
\hline & Mesh under support & Yes & No & Yes & No & No & No & No & No \\
\hline & Fragmentation & Yes & No & No & No & No & No & No & No \\
\hline & $\begin{array}{c}\text { 6LoWPAN } \\
\text { Header } \\
\text { Compression }\end{array}$ & Yes & $\begin{array}{c}\text { Yes } \\
\text { (star topol.) }\end{array}$ & $\begin{array}{c}\text { Yes } \\
\text { (address } \\
\text { adaptation) }\end{array}$ & $\begin{array}{c}\text { Yes } \\
\text { (star topology) }\end{array}$ & $\begin{array}{c}\text { Yes } \\
\text { (address } \\
\text { adaptation) }\end{array}$ & $\begin{array}{c}\text { Yes } \\
\text { (address } \\
\text { adaptation) }\end{array}$ & Yes & $\begin{array}{c}\text { Yes } \\
\text { (address } \\
\text { adaptation) }\end{array}$ \\
\hline & $\begin{array}{c}\text { 6LoWPAN } \\
\text { Neighbor Discovery }\end{array}$ & Yes & $\begin{array}{c}\text { Yes } \\
\text { (no multihop) }\end{array}$ & Yes & $\begin{array}{c}\text { Yes } \\
\text { (no multihop) }\end{array}$ & Partially & $\begin{array}{c}\text { With } \\
\text { DHCPv6 }\end{array}$ & $\begin{array}{l}\text { DHCPv6 } \\
\text { only }\end{array}$ & $\begin{array}{c}\text { Yes } \\
\text { (no multihop) }\end{array}$ \\
\hline & Multicast & L2 broadcast & L2 unicast & L2 broadcast & L2 unicast & L2 broadcast & L2 broadcast & No & L2 multicast \\
\hline & Privacy addresses & Not specified & Random IID & DHCPv6 & Random IID & Random IID & Random IID & DCHPv6 & Random IID \\
\hline & L2 security used & Yes & Yes & Yes & Yes & No & No & Yes & Yes \\
\hline
\end{tabular}

Table 1. Main features of IPv6-supported IoT technologies and their adaptation layers. L2 stands for Layer 2. 


\subsection{Routing}

IEEE 802.15.4 networks may follow a multihop topology. To support network connectivity in such a topology, 6LoWPAN offers two routing approaches: mesh-under, whereby routing is performed below IP (thus a multihop path appears as a single link to IP), and route-over, which relies on IP routing (thus each physical hop is an IP hop). In the latter, intermediate forwarders are IP routers called 6LoWPAN Routers (6LRs). In both approaches, the router that connects a 6LoWPAN network to another IP network is a 6LoWPAN Border Router (6LBR).

Most 6Lo technologies define network topologies whereby there is a single physical link between a host and the 6LBR. In these, a routing protocol is not needed. However, ITU-T G.9959 and IEEE 1901.2 support mesh networks. The 6Lo adaptation layers for these two technologies reuse 6LoWPAN route-over functionality (in both cases) or mesh-under (only in the former).

\subsection{Addressing}

In 6LoWPAN, as in more classic networking scenarios such as Ethernet, IPv6 address generation typically relies on embedding the link layer address in the Interface Identifier (IID) for stateless address autoconfiguration. In 6LoWPAN, this approach is also leveraged for total or partial address compression for IPv6 header compression. For the same reasons, all 6Lo adaptation layers initially assumed the same mechanism for IID generation. However, recent activity in the IETF, published in RFC 8065, has provided advice on privacy issues due to link-layer-address-based IIDs, including correlation of activities over time, location tracking or vendor-specific vulnerability exploitation. To mitigate such threats, IPv6 addresses should be frequently changed and should avoid the use of globally unique characteristics. To this end, different approaches to derive privacy IIDs or addresses have also been adopted for the 6Lo adaptation layers.

\subsection{Header compression}

When communicating an IPv6 packet over IEEE 802.15.4 or a 6Lo technology, a typical 40-byte IPv6 header would consume a significant fraction of frame payload, as well as precious energy and bandwidth resources. 6LoWPAN header compression (6LoWPAN HC) was defined to produce a lightweight encoding for the IPv6 header over IEEE 802.15.4. 6LoWPAN HC leverages stateless and stateful techniques. The former exploits the fact that some IPv6 header fields (e.g., IIDs, and datagram length) are derivable from the link layer header, and an optimistic expectation that several IPv6 header fields will be set to typical values. The latter requires 6LoWPAN nodes to share context that allows to substitute address prefixes or complete addresses by a short identifier. 
Subsequently, 6LoWPAN HC has served as the basis for header compression over the 6Lo technologies. 6LoWPAN HC has needed some tailoring to the specific characteristics of each technology, with the exception of IEEE 1901.2, which does not need any changes, since it endorses the IEEE 802.15.4 MAC frame and address formats.

The 6Lo specifications of IPv6 over ITU-T G.9959, MS/TP, NFC, and IEEE 802.11ah only introduce a minimal change to 6LoWPAN HC: the adaptation of the link layer address/identifier sizes in these technologies to the ones assumed in 6LoWPAN HC. For Bluetooth LE and DECT ULE, 6LoWPAN HC is more deeply modified. The star topology of these networks (see Figure 3) is exploited to optimize header compression. In such a topology, when the central device (a 6LBR) receives a packet from one of its neighboring hosts, it can infer that the host that sent the packet is its source. Likewise, when a host receives a packet from the central device, the host can derive that the host itself is the destination of the packet. Therefore, in the two described situations, the source or the destination IPv6 addresses can be fully omitted from the IPv6 header, respectively.

Another header compression mechanism suitable for 6LoWPAN- or 6Lo-based networks, which can be used as an addition to 6LoWPAN HC, is the 6LoWPAN Generic Header Compression (GHC). In contrast with 6LoWPAN HC, which is limited to IPv6 (and UDP) headers, GHC compresses headers of any kind, at the expense of a slight compression efficiency decrease.

\subsection{Fragmentation}

Most of the 6Lo/6LoWPAN target technologies exhibit a short maximum PHY/MAC data unit size, typically in the range between a few tens and a few hundreds of bytes (i.e. two to one orders of magnitude below the characteristic 1500-byte Maximum Transmission Unit (MTU) of Ethernet, respectively). This feature simplifies error control mechanisms, decreases processing and memory requirements, allows lower energy consumption and is suitable for the typically short-sized application-layer payloads in this space. However, IPv6 requires every Internet link to have a 1280-byte MTU. If the link layer technology supports fragmentation (and reassembly), such functionality can be exploited to overcome the problem. Otherwise, fragmentation must be performed at the 6LoWPAN/6Lo adaptation layer.

Among the set of 6LoWPAN/6Lo technologies, only IEEE 802.15.4 requires the use of adaptation layer fragmentation (which was defined by 6LoWPAN). As shown in Table 1 , the rest of technologies provide their own fragmentation, with the exception of MS/TP, which supports an MTU of 2032 bytes and thus allows transmitting unfragmented 1280-byte IPv6 packets. IEEE 802.11ah also supports long frames. Typical header/footer sizes and encapsulation of a large IPv6 datagram over each 6Lo/6LoWPAN technology are shown in Figure 1.b-1.c. 


\subsection{Encapsulation}

IPv6 datagram encapsulation over a 6Lo/6LoWPAN technology incurs overhead, which comprises headers and/or footers of physical, link, and adaptation layers. As shown in Figure 4, Bluetooth LE and DECT ULE exhibit the highest encapsulation overhead for medium and large size packets, due to their reduced link layer packet size, which leads to severe fragmentation. IEEE 802.15.4, ITU-T G.9959, IEEE 1901.2 and NFC experience moderate overhead increase for large packets. The encapsulation overhead is constant with the IPv6 datagram size for MS/TP ${ }^{1}$ and IEEE 802.11ah, which support an MTU greater than 1280 bytes.

Header compression reduces the number of IPv6 header transmitted bytes, and also helps decrease physical- and link-layer overhead, as it allows to reduce fragmentation. This is especially beneficial in Bluetooth LE and DECT ULE, where fragmentation can be avoided for short IPv6 datagrams. When fragmentation headers (either adaptation layer or link layer ones) are needed, their relative size is short in comparison with the other headers, as well as with the data payload size that requires fragmentation.

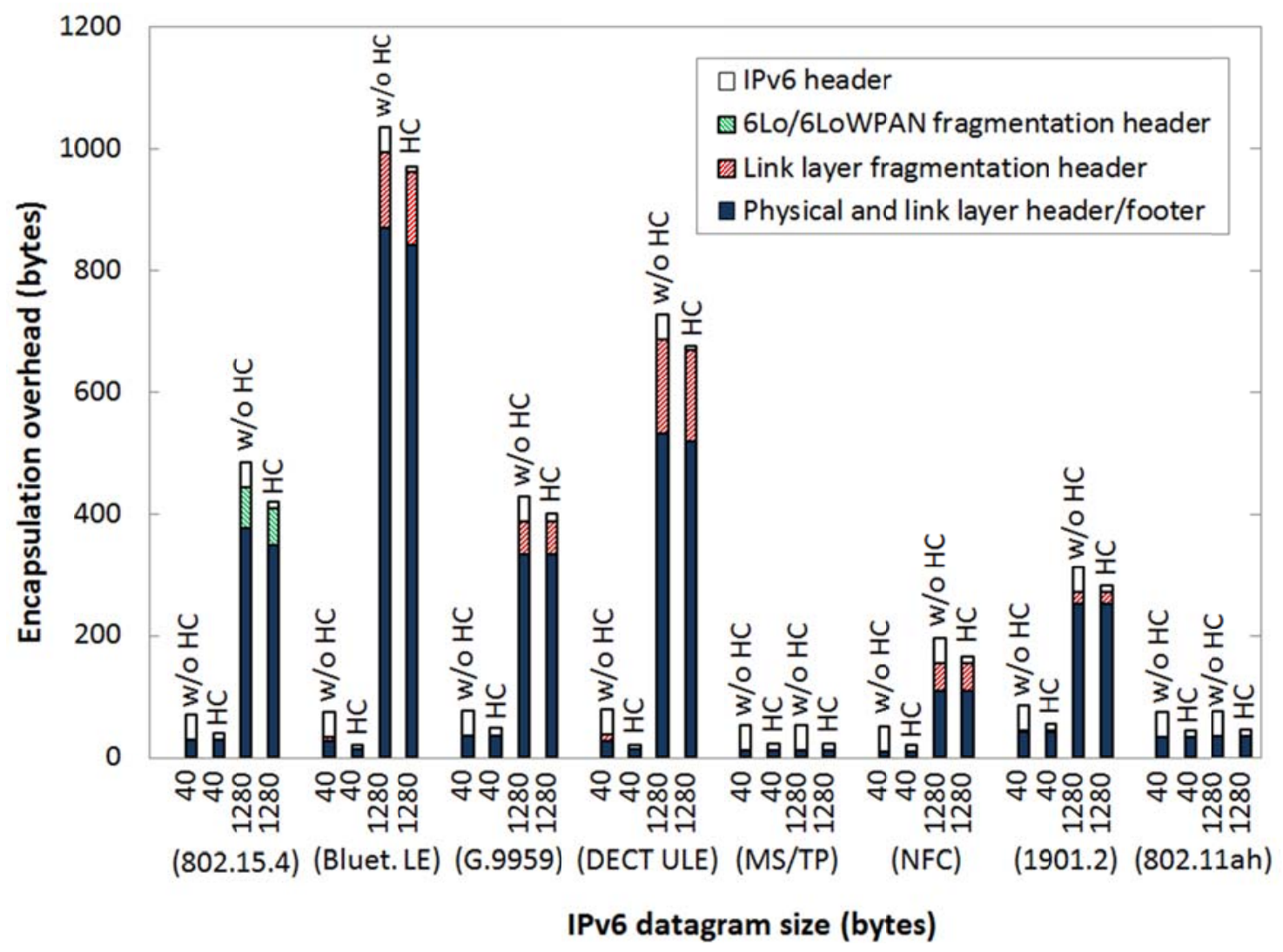

Figure 4. IPv6 datagram encapsulation and Header Compression (HC) overhead over IEEE 802.15.4 and the 6Lo technologies.

\footnotetext{
${ }^{1} \mathrm{MS} / \mathrm{TP}$ uses an encoding that allows to escape preamble sequences in other frame fields, which introduces an additional linear worst-case overhead of 1 in 254.
} 


\subsection{Neighbor discovery}

The IPv6 Neighbor Discovery (ND) protocol offers router and subnet prefix discovery, address resolution and duplicate address detection. ND was designed for transitive, high energy and bandwidth networking scenarios, where nodes' network interfaces are always on, and where the ND aggressive use of multicast is a good fit. While ND is useful to minimize human operational tasks (a good property for IoT environments), it is not well suited to constrained-node networks, which are often duty-cycled, offer nontransitive links, and map IPv6 multicast to network-wide broadcast. The optimized ND for 6LoWPAN (6LoWPAN ND), supports sleeping hosts by providing host-initiated interaction with routers, and minimizes the use of multicast. In addition, 6LoWPAN ND provides optional context dissemination for 6LoWPAN HC.

In 6Lo, the adaptation layer for each specific technology selects the most appropriate components of 6LoWPAN (or even classic) ND in each case, with the exception of IEEE 1901.2, which solely appears to rely on DHCPv6 for address autoconfiguration. Among the rest of 6Lo technologies, only ITU-T G.9959 supports mesh networks, and thus its 6Lo adaptation layer requires the use of 6LoWPAN ND multihop functionality. The 6Lo adaptation layer for MS/TP follows a different scheme, as for prefix discovery and address resolution it uses the classic IPv6 ND. The continuous power source of the devices, and the multidrop bus topology of MS/TP are a natural fit for this approach.

\subsection{Multicast}

An important consideration is how IPv6 multicast traffic is transported over a lower layer technology. Most 6Lo/6LoWPAN technologies support broadcast at the link layer, but not multicast. This is intrinsically inefficient since then the transmission of a multicast IPv6 packet may eventually lead to flooding a whole subnet. Among the considered technologies, only IEEE 802.11ah supports multicast in addition to broadcast at the link layer. The problem with IPv6 multicast aggravates over Bluetooth LE and DECT ULE. Bluetooth LE data channels only support unicast, while in DECT ULE the available MAC layer broadcast service is considered inadequate for IP multicast. Therefore, in these technologies, an IPv6 multicast packet may have to be unicast several times by the same node, e.g. when the 6LBR sends an IPv6 multicast packet to a subset of its neighbors. To mitigate the impact of this behavior on energy consumption and bandwidth, the 6LBR must only forward IPv6 multicast packets to nodes that have registered for the intended multicast group. 


\section{Challenges in $6 \mathrm{Lo}$}

This section discusses two main challenge areas for 6Lo: applicability and security.

\subsection{Applicability}

6LoWPAN was originally designed to enable IPv6 over IEEE 802.15.4 networks. Subsequently, 6Lo has adapted 6LoWPAN for other IoT technologies. Such adaptation can be successfully done when an intended IoT technology is relatively similar to, or less constrained than, IEEE 802.15.4. This is a very recent finding, as only the emerging category of IoT technologies called Low-Power Wide Area Networks (LPWAN) has evidenced the existence of 6Lo applicability limits.

LPWAN technologies offer long-range links (and thus low infrastructure cost) at the expense of extreme constraints that challenge IPv6 support. These comprise: i) MTU and bit rate up to several orders of magnitude below those in 6LoWPAN/6Lo technologies, ii) lack of layer-two fragmentation, iii) severe message rate limitations due to regulatory constraints, and iv) uplink/downlink asymmetry. Overall, transmission resources are drastically limited in relevant LPWAN technologies (Figure 5). For example, a flagship LPWAN technology called Sigfox typically offers uplink and downlink sustained capacities of $155 \mathrm{mbit} / \mathrm{s}$ (i.e., millibit per second!) and $3.7 \mathrm{mbit} / \mathrm{s}$, respectively. Other LPWAN technologies with similar constraints in some of their PHY/MAC options comprise LoRaWAN and IEEE 802.15.4k.

In order to enable IPv6 over such extraordinarily challenging scenarios, the level of adaptation provided by 6Lo is insufficient. Over LPWAN, 6LoWPAN/6Lo mechanisms for header compression, ND and fragmentation would all incur a too high overhead, even rendering deployments impractical in some cases. Therefore, a new IETF working group has been recently created to enable IPv6 over LPWAN technologies. While initial solution proposals are currently at an early development stage, there exists already a consensus to go significantly beyond 6Lo-style IPv6 adaptation for LPWAN. 


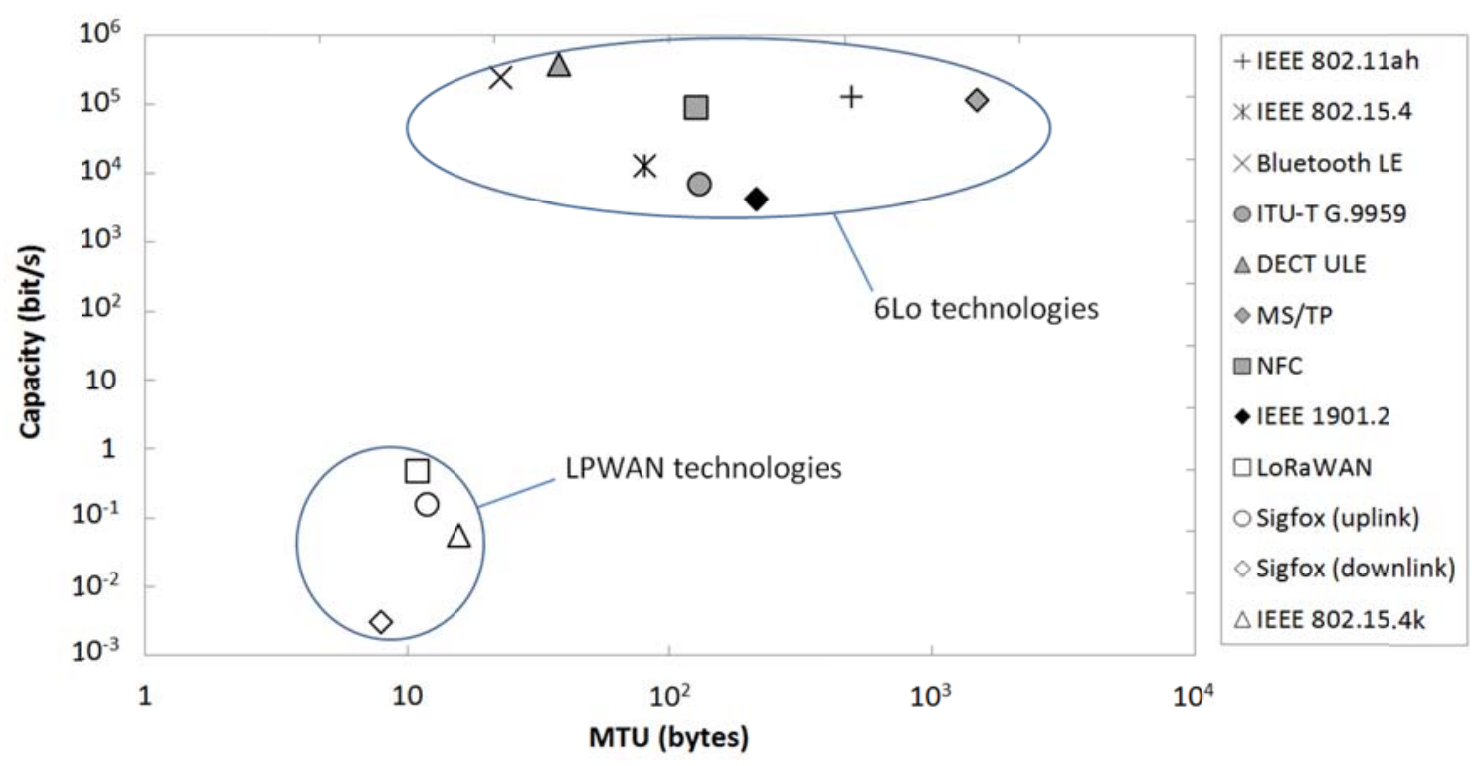

Figure 5. Clusters of 6Lo and LPWAN technologies in terms of their worst-case MTU and capacity.

\subsection{Security}

Security is a critical subject in IoT, since attacks in this area may compromise activities in a vast number of scenarios. End-to-end security mechanisms are available for constrained devices. On the other hand, 6Lo adaptation layers enforce use of link-layer security, which is supported by most 6Lo technologies. Exceptions comprise MS/TP, which is anyway harder to attack than a wireless technology, and NFC, which generally relies on its very short range for security. Both end-to-end and link-layer security mechanisms offer confidentiality, data integrity and authentication services. A major challenge in networks that employ link-layer security is avoiding double setup costs for both the link-layer and the end-to-end security required.

While 6Lo adaptation layers are required to support IPv6 over the 6Lo technologies, they also introduce additional security threats. Main 6Lo mechanisms exploitable by malicious entities comprise ND, fragmentation, and routing, as presented in the next subsections. Note that the related security challenges are solved by link-layer security as long as nodes do not become compromised. Otherwise, nodes may be subject to the threats and attacks described below.

\subsubsection{Neighbor Discovery}

An attacker may mount Denial of Service (DoS) attacks to ND. Examples include masquerading as a legitimate router by sending apparently correct ND messages, and flooding bogus traffic at a victim node. Countermeasures comprise prioritizing existing routers, and limiting the ND message rate, respectively. How a previously trusted router that becomes compromised can be handled is an open question. 


\subsubsection{Fragmentation/Reassembly}

A malicious node may periodically send a first fragment to reserve receiver reassembly buffer space for a relatively long time, causing other incoming fragments (from legitimate nodes) to be discarded by the receiver. An attacker may also transmit spoofed fragment duplicates, which lead to incorrect IPv6 packet reassembly. Countermeasures have been proposed [7], but have not been standardized.

\subsubsection{Routing}

A routing protocol, which is needed by some 6Lo technologies, may suffer brute-force attacks to disclosure and integrity of routing information. AES-CCM with a 128-bit key is considered a secure block cipher against such attacks. Further security measures comprise routing message rate limitation to avoid flooding DoS attacks, and path diversity to mitigate traffic analysis or traffic-discarding nodes. The main related open issue is key provisioning.

\section{Conclusion}

6Lo leverages 6LoWPAN to significantly increase the spectrum of IPv6-supported technologies. In comparison with 6LoWPAN, 6Lo adaptation layers tend to be more lightweight. Fragmentation at the 6Lo layer so far is not needed, while routing is required only for two 6Lo technologies. Another common feature of 6Lo adaptation layers is a customized use of 6LoWPAN neighbor discovery and header compression. The latter is crucial to reduce IPv6 datagram encapsulation overhead.

6Lo is only applicable for technologies relatively similar to IEEE 802.15.4 in terms of transmission capacity and MTU constraints. On the other hand, 6Lo introduces security challenges. Nevertheless, with a wide set of adaptation layers for a new generation of diverse technologies, 6Lo provides a fundamental cornerstone towards the 100-billion node Internet (of Things).

\section{Acknowledgments}

Carles Gomez has been funded in part by the Spanish Government through the Jose Castillejo grant CAS15/00336. His contribution to this work has been carried out in part during his stay as a visiting scholar at the Computer Laboratory of the University of Cambridge. Carles Gomez and Josep Paradells have been partially supported by the ERDF and the Spanish Government through project TEC2016-79988-P, AEI/FEDER, UE. The authors would like to thank the members of the IETF 6LoWPAN and 6Lo WGs. 


\section{References}

[1] A. Betzler, C. Gomez, I. Demirkol, J. Paradells, "CoAP Congestion Control for the Internet of Things", IEEE Communications Magazine, vol. 54, no. 7, Jul. 2016, pp. 154-160.

[2] Z. Sheng, S. Yang, Y. Yu, A.V. Vasilakos, J.A. Mccann, K.K. Leung, “A Survey on the IETF Protocol Suite for the Internet of Things: Standards, Challenges, and Opportunities", IEEE Wireless Communications Magazine, vol. 20, no. 6, Dec. 2013, pp. 91-98.

[3] D. Dujovne, T. Watteyne, X. Vilajosana, P. Thubert, "6TiSCH: Deterministic IP-Enabled Industrial Internet (of Things)", IEEE Communications Magazine, vol. 52, no. 12, Dec. 2014, pp. 36-41.

[4] J. Nieminen, C. Gomez, M. Isomaki, T. Savolainen, B. Patil, Z. Shelby, M. Xi, J. Oller, "Networking Solutions for Connecting Bluetooth Low Energy Enabled Machines to the Internet of Things", IEEE Network Magazine, vol. 28, no. 6, Nov.-Dec. 2014, pp. 83-90.

[5] C. Gomez, J. Paradells, "Wireless Home Automation Networks: A Survey of architectures and technologies", IEEE Communications Magazine, vol. 48, no. 6, Jun. 2010, pp. 92-101.

[6] C. Bormann, A. Castellani, Z. Shelby, "CoAP: An Application Protocol for Billions of Tiny Internet Nodes", IEEE Internet Computing, vol. 16, no. 2, Mar. 2012, pp. 62-67.

[7] J. Granjal, E. Monteiro, J.S. Silva, "Security for the Internet of Things: A Survey of Existing Protocols and Open Research Issues", IEEE Communications Surveys \& Tutorials, vol. 17, no. 3, Aug. 2015, pp. 1294-1312.

\section{Biographies}

Carles Gomez received his Ph.D. degree from Universitat Politècnica de Catalunya in 2007. He is an associate professor at the same university. He is a co-author of numerous technical contributions including papers published in journals and conferences, IETF RFCs, and the book "Sensors Everywhere - Wireless Network Technologies and Solutions". His current research interests focus mainly on the Internet of Things. He serves as Associate Editor of the Journal of Ambient Intelligence and Smart Environments.

Josep Paradells is a professor at Universitat Politècnica de Catalunya and director of the i2CAT Foundation. He has participated in national and European publicly funded 
research projects, and collaborated with the main Spanish telecommunications companies. He has published his research results in conferences and journals. His expertise area is wireless Internet combining theoretical studies with real implementations. His main focus is IoT, radio wake-up, and visible light communication.

Carsten Bormann is Honorarprofessor for Internet technology at the Universität Bremen and a board member of its Center for Computing and Communications Technology (TZI). He is a protocol designer by heart, a standardization geek by necessity, and a coauthor of the book entitled "6LoWPAN: The Wireless Embedded Internet" (Wiley, 2009).

Jon Crowcroft has been the Marconi Professor of Communications Systems in the Computer Laboratory, University of Cambridge since October 2001. He has worked in the area of Internet support for multimedia communications for over 30 years. Three main topics of interest have been scalable multicast routing, practical approaches to traffic management, and the design of deployable end-to-end protocols. Current active research areas are opportunistic communications, social networks, and scaling infrastructure-free mobile systems. 
a)

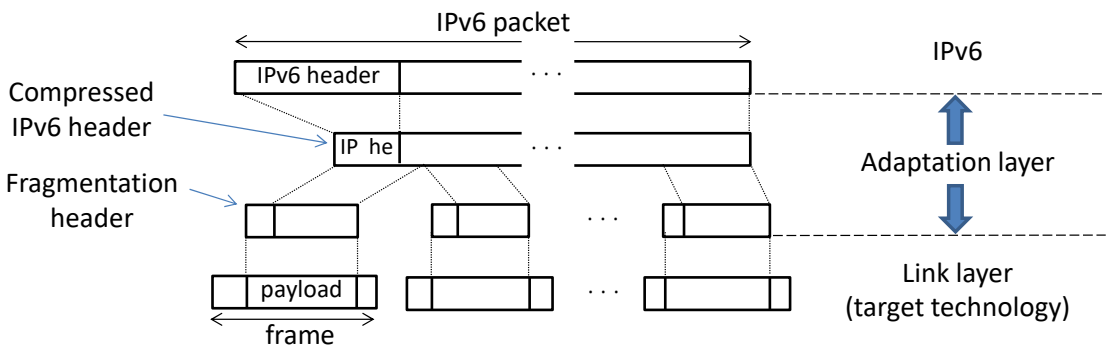

b)
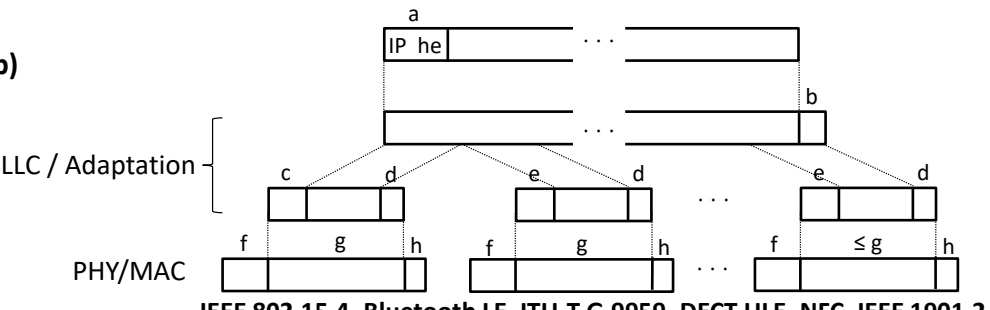

IEEE 802.15.4, Bluetooth LE, ITU-T G.9959, DECT ULE, NFC, IEEE 1901.2

c)

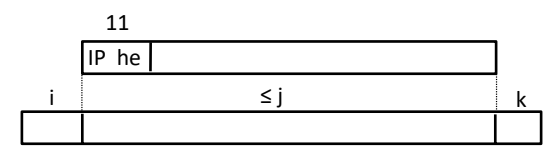

PHY/MAC

MS/TP, IEEE 802.11ah

\begin{tabular}{|c|c|c|c|c|c|c|}
\cline { 2 - 7 } \multicolumn{1}{c|}{} & \multicolumn{7}{c|}{ Field size (bytes) } \\
\hline Field & IEEE 802.15.4 & Bluetooth LE & ITU-T G.9959 & DECT ULE & NFC & IEEE 1901.2 \\
\hline a & 11 & 7 & 11 & 7 & 11 & 11 \\
\hline b & 0 & 0 & 0 & 2 & 0 & 0 \\
\hline c & 4 & 8 & 4 & 4 & 7 & 0 \\
\hline d & 0 & 0 & 2 & 0 & 0 & 0 \\
\hline e & 5 & 6 & 5 & 4 & 7 & 0 \\
\hline f & 29 & 15 & 34 & 12 & 5 & 40 \\
\hline g & 102 & 27 & 158 & 38 & 128 & 215 \\
\hline h & 2 & 3 & 2 & 2.5 & 2 & 2 \\
\hline
\end{tabular}

\begin{tabular}{|c|c|c|}
\cline { 2 - 3 } \multicolumn{1}{c|}{} & \multicolumn{2}{c|}{ Field size (bytes) } \\
\hline Field & MS/TP & IEEE 802.11ah \\
\hline $\mathbf{i}$ & 8 & 30 \\
\hline j & 1500 & 7951 \\
\hline $\mathbf{k}$ & 5 & 4 \\
\hline
\end{tabular}

Figure 1.a) An example of adaptation layer functionality; b) IPv6 datagram encapsulation over 6 Lo technologies, fragmentation needed; c) IPv6 datagram encapsulation over 6Lo technologies, fragmentation not needed. 


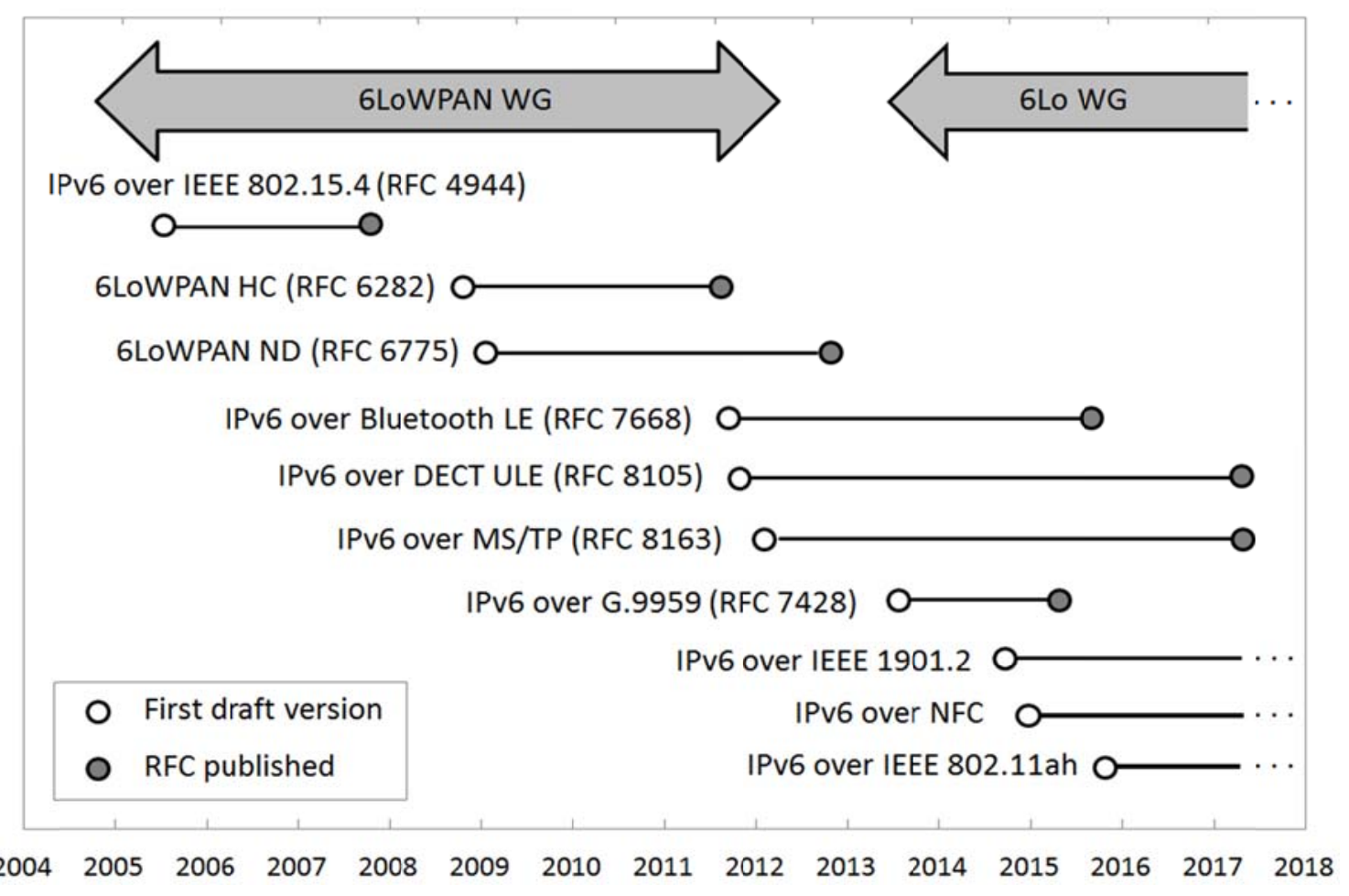

Figure 2. Chronology and timespan of the 6LoWPAN and 6Lo WGs adaptation layer specifications and activity. 


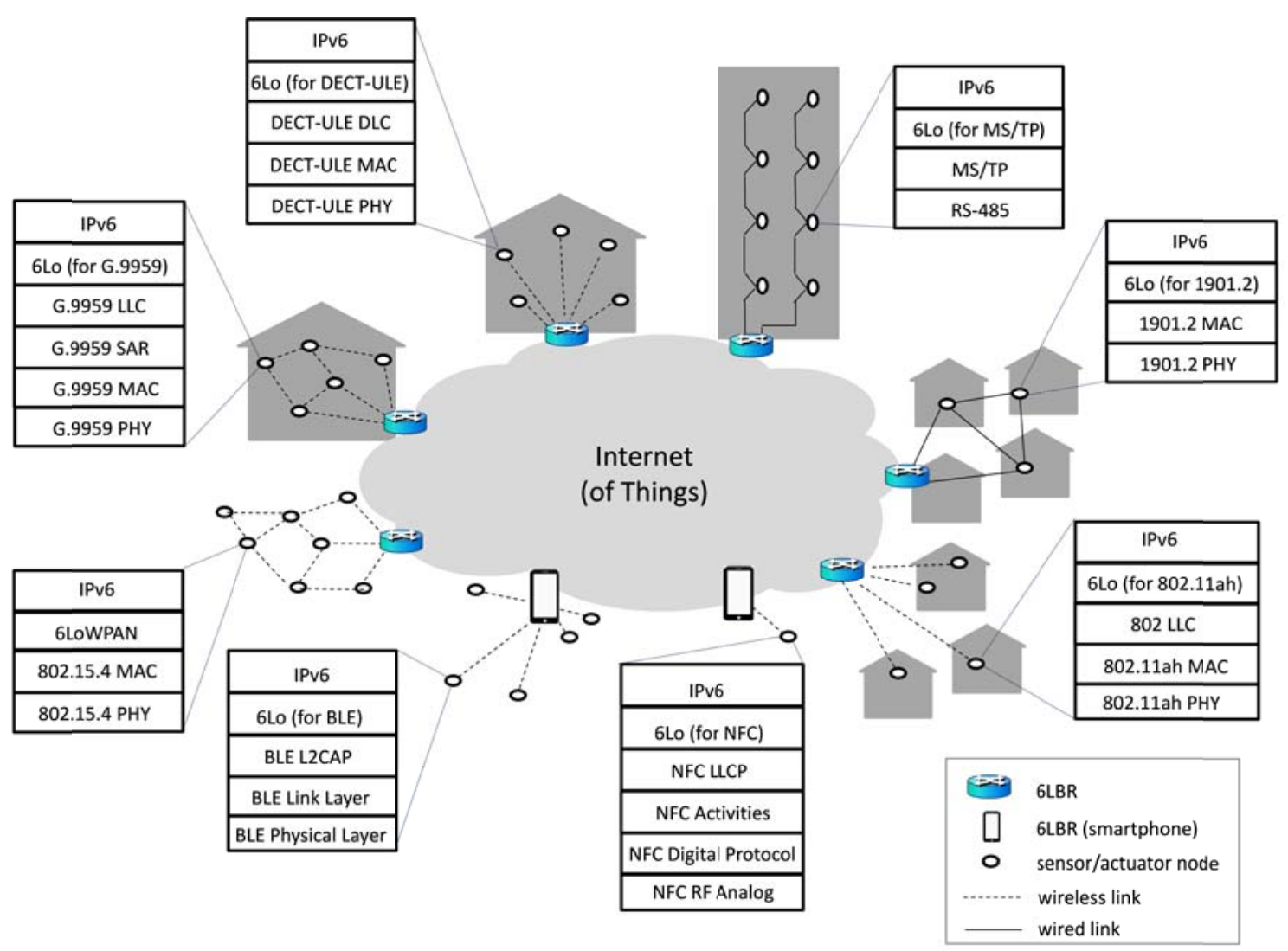

Figure 3. Protocol stack, and example network topology, for each 6Lo/6LoWPAN technology when using IPv6. BLE stands for Bluetooth LE. 


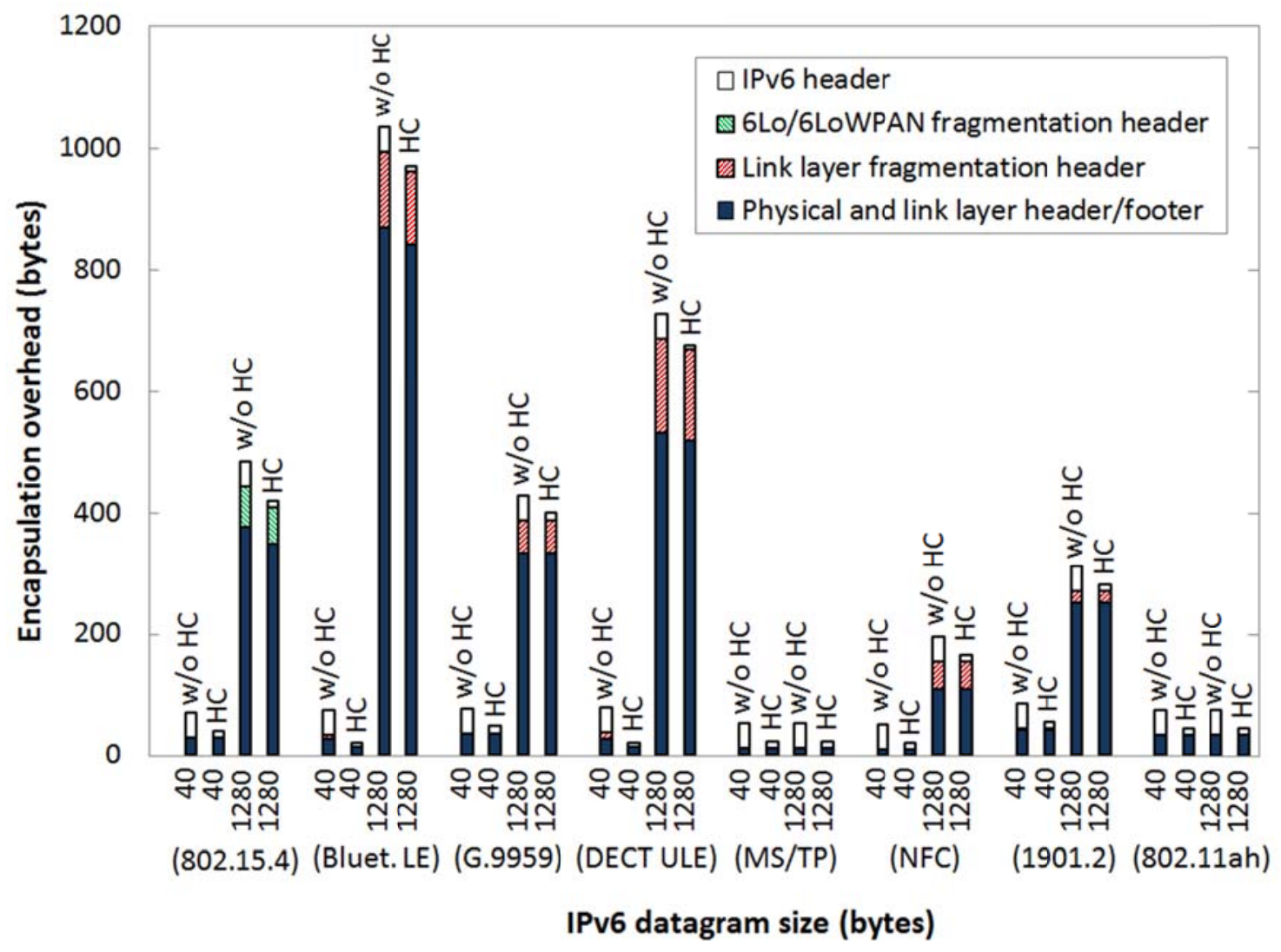

Figure 4. IPv6 datagram encapsulation and Header Compression (HC) overhead over IEEE 802.15.4 and the 6Lo technologies. 


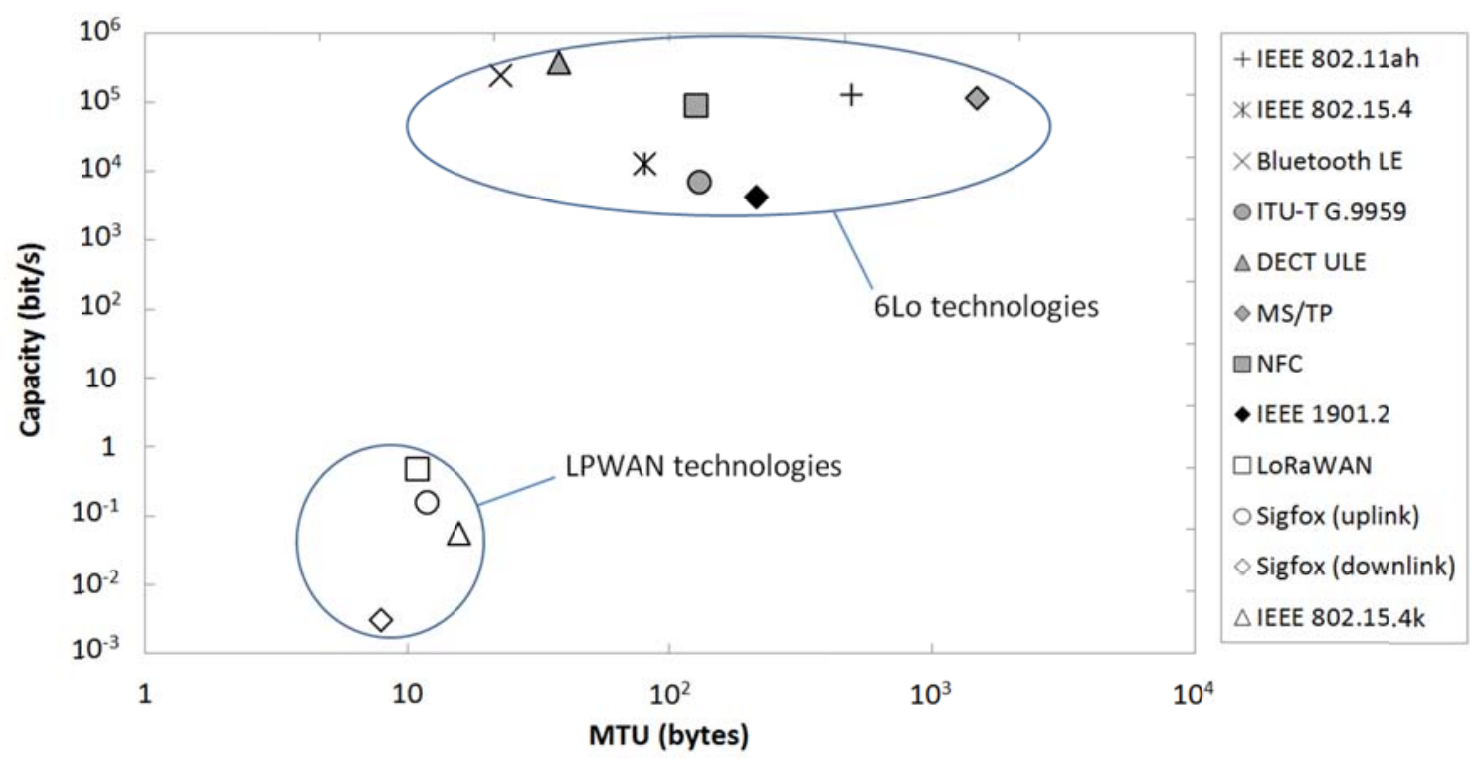

Figure 5. Clusters of 6Lo and LPWAN technologies in terms of their worst-case MTU and capacity. 


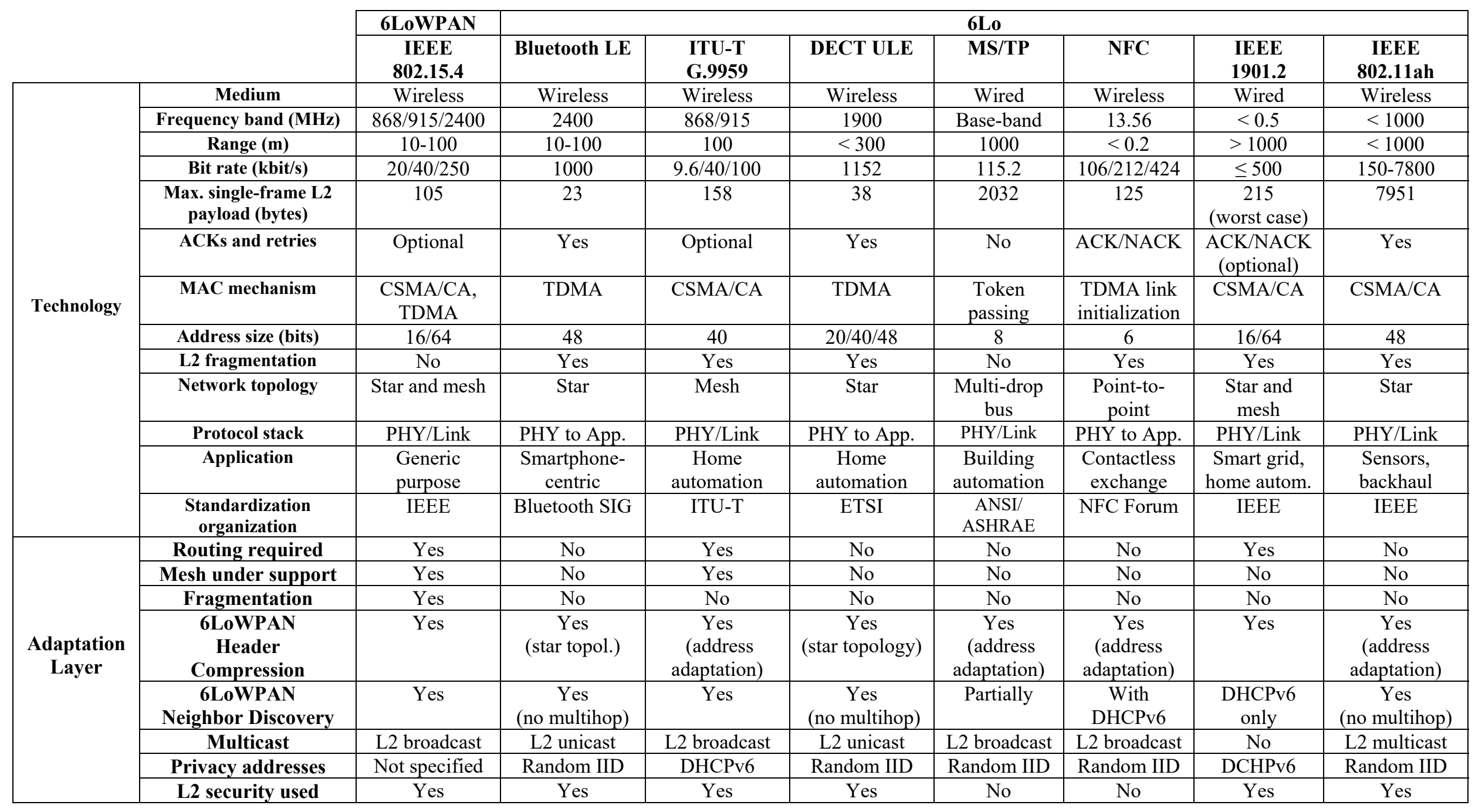

Table 1. Main features of IPv6-supported IoT technologies and their adaptation layers. L2 stands for Layer 2. 